\title{
Twinning in nanocrystalline Ni by severe plastic deformation
}

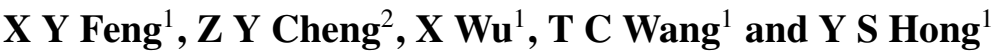 \\ ${ }^{1}$ State Key Laboratory of Nonlinear Mechanics, Institute of Mechanics, Chinese Academy of \\ Sciences, Beijing 100080, People's Republic of China \\ ${ }^{2}$ Department of Materials Science, Tsinghua University, Beijing, People's Republic of China \\ E-mail: xlwu@mech.ac.cn
}

Received 22 October 2005, in final form 3 January 2006

Published 3 February 2006

Online at stacks.iop.org/JPhysD/39/746

\begin{abstract}
Deformation twinning is confirmed upon large plastic deformation in nanocrystalline (nc) Ni by transmission electron microscopy examinations. New and compelling evidence has been obtained for several twinning mechanisms that operate in nc grains, with the grain boundary emission of partial dislocations determined as the most proficient. Twinning in nc Ni may be interpreted in terms of molecular dynamics simulation based on generalized planar fault energy curves.
\end{abstract}

\section{Introduction}

Nanocrystalline (nc) metals possess superior mechanical properties such as high strength, which sometimes coexist with very good ductility $[1,2]$. These exceptional properties are attributed to their unique deformation mechanisms [3,4], which are fundamentally different from those in their grained counterparts. In grains with diameters in the range of several tens of nanometres, a partial dislocation mediated process is predicted to be a dominant deformation mechanism from molecular dynamics (MD) simulation [5-7]. For nc FCC metals, in particular, deformation twinning (DT) via partial dislocation emission from the grain boundary (GB) as a contributing plastic deformation mechanism has been evidenced by either experimental observation in $\mathrm{Al}$ [8-10], $\mathrm{Cu}$ [11] and $\mathrm{Pd}$ [12] or MD simulation in $\mathrm{Al}$ [13]. These observations are surprising because DT has never been observed in their coarse-grained counterparts under normal conditions. Recently, Swygenhoven and co-workers [14,15] revealed by a series of MD the detailed mechanism behind DT in terms of the generalized planar fault (GPF) energy curves. They showed that DT is not favoured over isolated extended partial or full dislocation activity during uniaxial tension in three nc FCC metals, Ni, Cu and Al, with substantially different GPF energy curves. More recently, an in situ x-ray diffraction study demonstrated no evidence of DT and a full dislocation event as a deformation mechanism during tensile deformation of nc Ni [16]. On the other hand, however, there may not be enough time in the simulation timescale for the trailing partial or the partial in an adjacent plane to be emitted for forming a full dislocation or a twin [14]. Moreover, the deformation mechanism is not the same for all FCC metals based on MD simulation [14], independent of the absolute value of the stacking fault (SF) energy. Recently, an analytical model was developed to predict the nucleation and growth of deformation twins in nc metals $[17,18]$. On the experimental side there has not been any report till date showing direct evidence of whether or not DT is possible in $\mathrm{nc} \mathrm{Ni}$. In this paper, we will provide such information by illustrating DT and its mechanisms based on TEM observations in $\mathrm{nc} \mathrm{Ni}$, for the purpose of elucidating the deformation mechanism in $\mathrm{nc} \mathrm{Ni}$.

\section{Experimental}

A high-purity polycrystalline Ni (purity: $99.998 \mathrm{wt} \%$ ) was used in this study. The average grain size was determined to be $\sim 40 \mu \mathrm{m}$. The shape of the specimen was $100 \times 100 \times 8 \mathrm{~mm}^{3}$.

The nc Ni was obtained by the surface mechanical attrition treatment (SMAT) technique [19]. The details of the SMAT process have been given in our previous papers [19-21]. In brief, during the SMAT process, the hardened steel balls (shots) $8 \mathrm{~mm}$ in diameter were placed at the bottom of a cylindershaped vacuum chamber attached to a vibration generator, with which the balls were resonated. Because of the high vibration frequency of the system, the sample surface to be treated was peened repetitively by a large number of balls within a short period of time. Each peening of the ball to the surface resulted in plastic deformation in the surface layer 


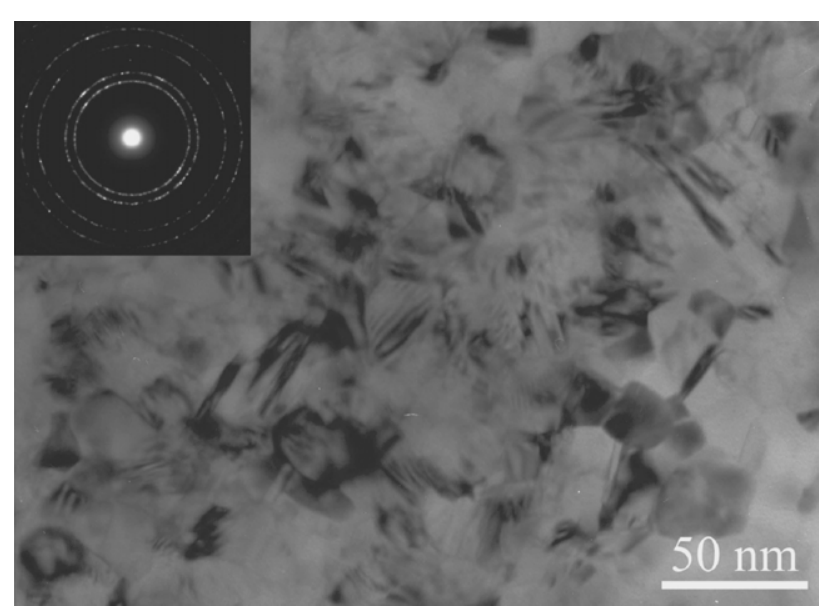

Figure 1. TEM micrograph showing the nc grains of $\mathrm{Ni}$ after mechanical attrition. In the inset is the SAED pattern.

of the treated sample. As a consequence the grains in the surface layer were expected to be effectively refined. In the present work, the SMAT process was performed for $50 \mathrm{~min}$ at room temperature with a vibrating frequency of $50 \mathrm{~Hz}$ under vacuum. As for the deformed material, both strain and strain rate had a gradient variation from the treated surface (both are extremely large) towards the deep matrix (essentially zero), and, as a result, the grain sizes with gradient distribution were developed in the treated sample [19-21]. Hence, one may examine the microstructure characteristics at different levels of strain to reveal the underlying mechanism of microstructural evolution within various grain size regimes.

Following the SMAT, the microstructural characterization was performed in a field emission gun high-resolution transmission electron microscope (HRTEM). The thin foils for HRTEM observations were prepared by means of cutting, grinding, dimpling and a final ion thinning or twin-jet polishing at low temperature.

\section{Results}

After SMAT the nc grains $(<100 \mathrm{~nm}$ in size $)$ are present from a depth of $\sim 30 \mu \mathrm{m}$ to the top surface of the treated layer. Figure 1 displays a plan view TEM micrograph of the deformation microstructure of $\mathrm{Ni}$ ( $\sim 20 \mu \mathrm{m}$ deep $)$. Most grains are equiaxed with a mean grain size $\sim 50 \mathrm{~nm}$. In the inset is the electron diffraction pattern. The pattern exhibits rings, indicating highangle GBs (HiAGBs) and random crystallographic orientations in the selected field of view.

Figure 2(a) shows two nc grains labelled A and B, respectively. Figure $2(b)$ is the enlarged view of the outlined area in figure $2(a)$. A deformation twin, T1, is visible at the lower part of grain A. The arrowhead A indicates the twin boundary (TB) starting from the GB. The whole lower part below the TB has been transformed into a twin. For grain B, similarly, the upper part has also been transformed into a deformation twin, T2, with its TB marked by the arrowhead B. The insets are the fast Fourier transformations of the region that encompass the $\mathrm{A} / \mathrm{T} 1$ and $\mathrm{B} / \mathrm{T} 2$, respectively. These twins were likely heterogeneous nucleates at the corner of the grain and grow via the emission of Shockley partial dislocations from GBs $[9,10,13]$.

In particular, a microtwin together with a $\mathrm{SF}$ is visible from the inset along the T1-A TB indicated by arrowhead A. The microtwin has a thickness of two atomic planes. Such a twin is formed by absorbing extended partial dislocations from neighbouring planes, i.e. via dynamic overlapping of SFs on adjacent planes [13]. Such a mechanism occurs when the concentration of the extended dislocations inside the grain is rather high. This mechanism explains the formation of twins in a homogenous fashion inside a grain.

It is further interesting to note the curved $\mathrm{TB}$ a-b between $\mathrm{T} 1$ in grain $\mathrm{A}$ and $\mathrm{T} 2$ in grain $\mathrm{B}$. This $\mathrm{TB}$ consists of many straight coherent segments (examples are marked by straight lines), arranged in a parallel and orderly way, which are connected by incoherent, non-crystallographic segments. T1 starts from around a, and broadens towards the left into A while it lengthens, whereas T2 grows in the opposite direction starting from $b$. As confirmed in the fast Fourier transformation (inset), the two grains (A and $\mathrm{B}$ ) happen to be in a twin relationship. The twin formation in each grain renders $\mathrm{T} 1$ and $\mathrm{T} 2$ in twin relationship as well. This leads to a curved TB when the two growing twins ( $\mathrm{T} 1$ and $\mathrm{T} 2$ ) meet. In addition, the T2-B TB is also waved due to a dislocation pile-up near TB marked by Ts. This indicates that the TB blocks the motion of dislocations during plastic deformation.

Figure 3 is a HRTEM image of an $\mathrm{nc} \mathrm{Ni}$ grain. Note that the TB, A1-A2, is a part of a HiAGB marked with asterisks. The A2-C-B nearby is a HiAGB, which is a part of a low-angle GB (LoAGB) that extends beyond B. Also note the presence of another TB, A1-B. These features are reminiscent of the GB splitting mechanism shown by the MD simulation [13], the subsequent migration of a GB segment leaves behind two coherent TBs. Specifically, twinning takes place via the dissociation of an initial segment of the HiAGB into a TB (A1-A2) and a new HiAGB. The TB remains at the same position as before the dissociation, while the migration of the HiAGB segment to the new (A2-B-C) location produces the twin lamellae (A1-B-C-A2-A1), which is separated from the non-twinned lattice by a second TB (A1-B). That A2$\mathrm{C}-\mathrm{B}$ coincides with a pre-existing LoAGB suggests that the migrating HiAGB is eventually blocked by the LoAGB.

Figure 4 is the statistical distribution of the grain size showing deformation twins in $29 \mathrm{nc}$ grains. The wide distribution of grain sizes is present for twinning to occur, but most observations of twins are in grains ranging from 25 to $45 \mathrm{~nm}$ in size.

\section{Discussion and conclusions}

The MD simulation describes the physical insight into DT behind three kinds of partial dislocation activity during plastic deformation of nc FCC metals, with the GB acting as a source and sink of dislocations [14]. After nucleating a Shockley partial dislocation from the GB, if no other partial is emitted from the GB, the initial single partial will travel across the entire grain cross-section to be absorbed in an opposite GB leaving behind an extended SF. If this single partial dislocation is, however, followed by the nucleation of a trailing partial dislocation at the same slip plane, a full dislocation is formed. 
(a)

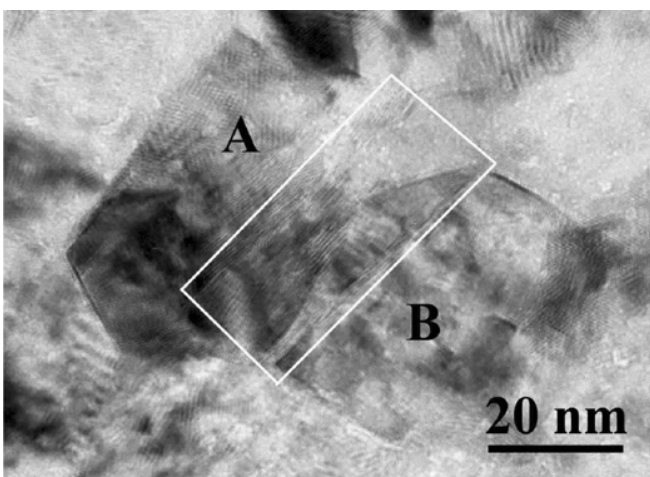

(b)

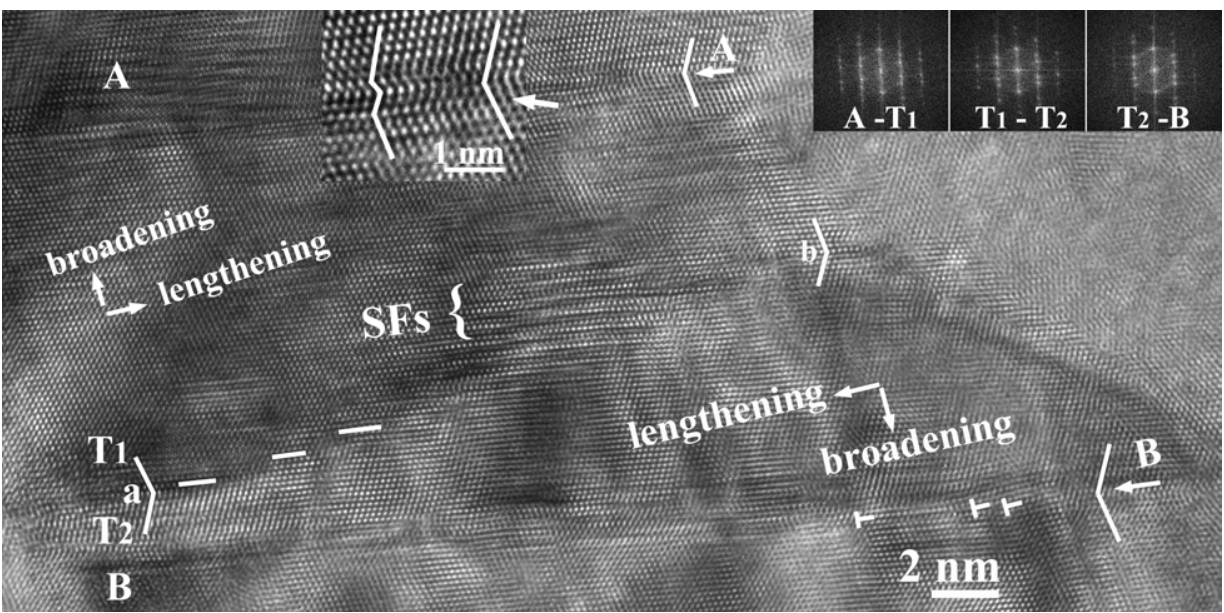

Figure 2. (a) Low magnification TEM image showing nc grains A and B. (b) A HRTEM micrograph showing growing twins, T1 in grain A and T2 in grain B, which lengthen and broaden and eventually meet to form a curved TB, a-b. A cluster of SFs is also observed nearby. The twin relationship between grains $\mathrm{A}$ and $\mathrm{B}$, and that between $\mathrm{A} / \mathrm{T} 1, \mathrm{~B} / \mathrm{T} 2$, and T1/T2, are confirmed in the fast Fourier transformation of the respective regions. Note the microtwin formation along the T1/A TB by dynamic overlapping of extended partial dislocations, i.e. absorbing SFs on adjacent slip planes. The T2/B TB is wavy as it is decorated by dislocations.

If the trailing partial is emitted onto the adjacent slip plane, a microtwin is formed. The interpretation of the partial dislocation mediated plasticity requires the GPF energy curve, which entails considering the stable and unstable SF energy, and unstable twin fault energy, namely $\gamma_{\mathrm{sf}}$ and $\gamma_{\mathrm{usf}}$ and $\gamma_{\mathrm{utf}}$, respectively [14]. For Ni, the energy barrier for twin formation, $\gamma_{\mathrm{utf}}-\gamma_{\mathrm{sf}}$, is higher than the barrier for a full dislocation $\gamma_{\text {usf }}-\gamma_{\text {sf }}$. Once a partial has been nucleated, full dislocation and twin fault events require the overcoming of a relatively high energy barrier (refer to the GPF curves of Ni in [14]). Hence, only extended SFs are generally observed in the simulation of $\mathrm{Ni}$ and both twinning and full dislocation activities are rarely seen in $\mathrm{Ni}[14]$.

In our case, however, twinning occurs after the mechanical attrition-a severe deformation method providing high shear stresses [19]. The GBs obtained by severe deformation is in a high non-equilibrium state $[22,23]$. The local high stress may be built up at GBs. Therefore, when $\mathrm{nc} \mathrm{Ni}$ is forced to deform to large strains, there will inevitably be a build-up of local stress concentrations in the deforming nanostructure that are at sufficient levels for twinning to become a competitive deformation response. Experiments have shown that when high stresses are provided (as in the case during ball milling $[9,10]$ and high-pressure torsion [11]), twinning is observed as a deformation mechanism. Simulation has demonstrated that the local stress is a crucial factor in the determination of the type of dislocation activity $[14,15]$. When applying a larger than normally-used stress to the simulated nc sample, not only is there a significant increase in the amount of dislocation activity, but in this stress regime all three slip mechanisms are seen and multiple twinning events are seen for $\mathrm{Ni}$ [15]. In other words, if the stress in the GB is kept high enough, full dislocations and/or twins might be observed, by means of a partial dislocation mediated process. Note that all the experimental twinning evidence in nc FCC metals so far are for deformation conditions that result in high deformation stresses, owing to high strain rates and/or large plastic deformation leading to saturated defect levels, such as indentation [8], grinding [8], high-pressure torsion [11], highrate cold rolling [12], and ball milling [9, 10,24] (sometimes with powders immersed in liquid nitrogen).

In summary, DT may occur in nc Ni after mechanical attrition. Three twinning mechanisms are observed including heterogeneous twins nucleated from GB, twin lamellae via the dissociation and migration of GB and dynamic overlapping of SF ribbons formed by Shockley partial dislocations emitted from the GB. DT becoming probable at high stresses and relatively large plastic strains are in fact exactly what is predicted by MD simulations based on GPF energy curves for nc FCC metals. In addition, among the several possible 


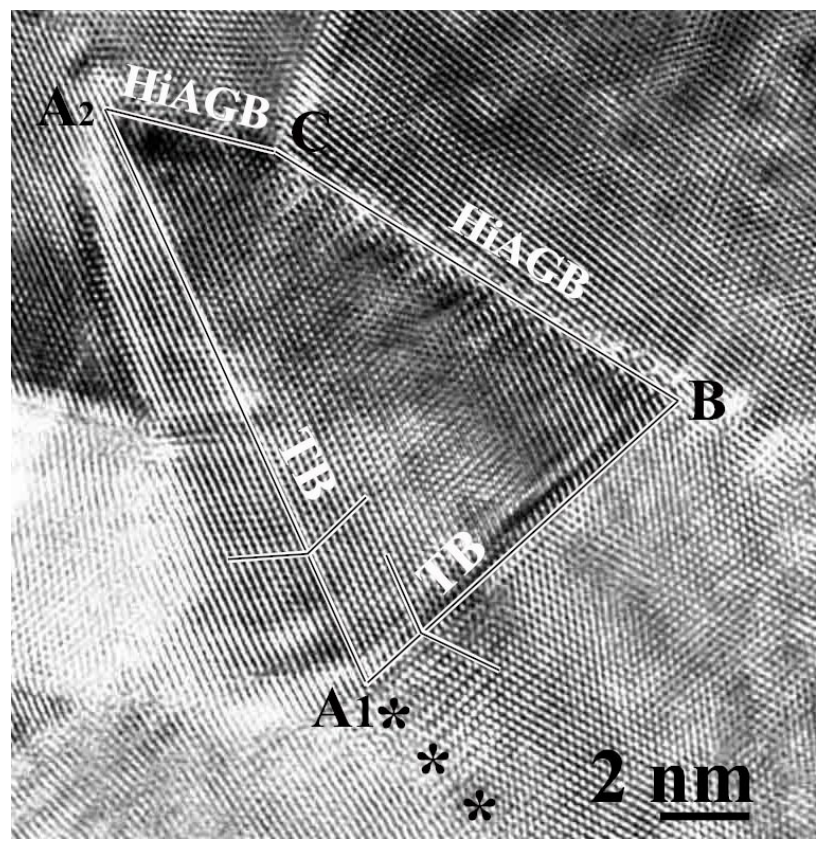

Figure 3. A HRTEM image of the formation of twin lamellae and its boundaries can be explained based on the GB splitting and migration mechanism. The labels and the evolution of the boundaries are explained in the text.

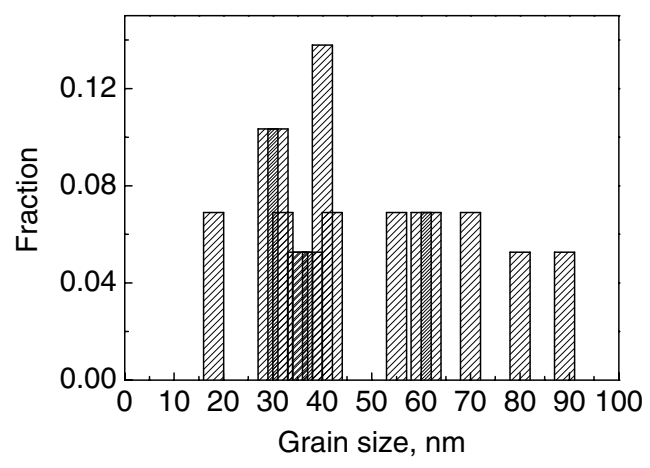

Figure 4. The statistical distribution of the grain size with deformations for twinning to occur.

mechanisms that have been identified, our survey of about 30 grains containing deformation twins led us to conclude that heterogeneous formation through GB emission of partial dislocations is the most active mechanism responsible for twinning in nc grains of $\mathrm{Ni}$.

\section{Acknowledgments}

This work was supported by the National Natural Science Foundation of China (Grant Nos 50471086, 50571110, 10472117 and 50021101),the national 973 Program of China (Grant No 2004CB619305), the Hong Kong Polytechnic University (Grant no BB90) and the National Center for Nanoscience and Technology, China.

\section{References}

[1] Wang Y M, Chen M W, Zhou F H and Ma E 2002 Nature 419 912

[2] Valiev R Z, Alexandrov I V, Zhu Y T and Lowe T C 2002 J. Mater. Res. 185

[3] Van Swygenhoven H 2002 Science 29666

[4] Ovid'ko I A 2002 Science 2952386

[5] Van Swygenhoven H, Spaczer M, Caro A and Farkas D 1999 Phys. Rev. B 6022

[6] Yamakov V, Wolf D, Phillpot S R and Gleiter H 2003 Acta Mater. $\mathbf{5 1} 4135$

[7] Schiotz J, Tolla F D and Jacobsen K W 1998 Nature 391561

[8] Chen M W, Ma E, Hemker K J, Sheng H W, Wang Y M and Cheng X 2003 Science 3001275

[9] Liao X Z, Zhou F, Lavernia E J, He D W and Zhu Y T 2003 Appl. Phys. Lett. 83632

[10] Liao X Z, Zhou F, Lavernia E J, He D W and Zhu Y T 2003 Appl. Phys. Lett. 835062

[11] Liao X Z, Zhao Y H, Srinivasan S G, Zhu Y T, Valiev R Z and Gunderov D V 2004 Appl. Phys. Lett. 84592

[12] Rosner H, Markmann J and Weissmuller J 2004 Phil. Mag. Lett. 84321

[13] Yamakov V, Wolf D, Phillpot S R, Mukherjee A K and Gleiter H 2004 Nature Mater. 343

[14] Van Swygenhoven H, Derlet P M and Frøseth A G 2004 Nature Mater. 3399

[15] Frøseth A G, Derlet P M and Van Swygenhoven H 2005 Adv. Eng. Mater. 716

[16] Budrovic Z, Van Swygenhoven H, Derlet P M, Van Petegem S and Schmitt B 2004 Science 304273

[17] Zhu Y T, Liao X Z, Zhao Y H, Srinivasan S G, Zhou F and Lavernia E J 2004 Appl. Phys. Lett. 855049

[18] Zhu Y T, Liao X Z, Srinivasan S G and Lavernia E J 2005 J. Appl. Phys. 98034319

[19] Lu K and Lu J 1999 J. Mater. Sci. Technol. 15193 Lu K and Lu J 2004 Mater. Sci. Eng. A 375-377 38

[20] Wu X, Tao N, Hong Y, Xu B, Lu J and Lu K 2002 Acta Mater. $\mathbf{5 0} 2075$

[21] Wu X, Tao N, Hong Y, Liu G, Xu B, Lu J and Lu K 2005 Acta Mater. $\mathbf{5 3} 681$

[22] Valiev R Z, Mishral R, Grozal J and Mukherjee A 1996 Scr. Mater. 341443

[23] Wu X, Tao N, Hong Y, Lu J and Lu K 2005 J. Phys. D: Appl. Phys. 384140

[24] He J H and Lavernia E J 2001 J. Mater. Res. 162724 